\title{
Bases filosóficas de la organización de la información
}

\author{
Miguel Angel Rendon Rojas
}

\author{
Investigador del Centro Universitario de \\ Investigaciones Bibliotecológicas de la \\ Universidad Nacional Autónoma de México-UNAM. \\ Doctor em filosofia
}

\section{Lizbeth Berenice Herrera Delgado}

\begin{abstract}
Licenciada en Bibliotecología por la Facultad de Filosofía y Letras de la Universidad Nacional Autónoma de México. studiante de Maestría en el Programa de Posgrado de Bibliotecología y Estudios de la Información de la UNAM
\end{abstract}

El orden, como categoría filosófica parte de la cosmovisión griega y es uno de los principios de la organización de la información. Se analiza la naturaleza del orden, su origen y su presencia en los sistemas de clasificación bibliotecológicos. Se concluye que ese orden es introducido por el bibliotecólogo, pero sin tener consecuencias subjetivistas-relativistas, el "cosmos documental" construido tiene fundamentos ontológicos objetivos emanados del "ser informacional" del sujeto, por lo que posee regularidades y características que pueden ser estudiadas objetivamente.

Palabras claves: Epistemología de la Bibliotecología; Organización de la información; Organización bibliográfica; Orden bibliográfico; Sistemas de clasificación; Orden.

\section{Bases filosóficas da organização da informação}

A ordem, como categoria filosófica, provem da filosofia grega e é um dos princípios da organização da informação. Analisamos a natureza da ordem, de sua origem e de sua presença nos sistemas da classificação da ciência da informação. Nós concluímos que esta ordem é introduzida pelo bibliotecário, mas sem consequências subjetivistas ou relativistas. O "documentário cosmo" 
construído tem bases ontológicas objetivas procedentes do "ser informativo" do sujeito, por isso tem regularidades e características que podem ser estudadas objetivamente.

Palavras-chave: Epistemologia da ciência da informação; Organização da informação; Organização bibliográfica; Ordem bibliográfica; Sistemas da classificação; Ordem.

\section{Philosophical foundations of the organization of information}

Order, as a philosophical category, comes from the Greek philosophy and is one of the principles of information organization. The nature of order as well as of its origin and its presence within the classification systems of Information Science is analyzed. We conclude that this order is introduced by librarians without, however, subjective or relativistic consequences. The constructed "documentary cosmos" has objective ontological basis from the "informational being" of the subject; thus, it has regularities and characteristics that can be objectively studied.

Keywords: Epistemology of Library Science; Organization of information; Bibliographical Organization; Bibliographical Order; Classification systems; Order.

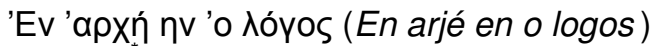
Jn. $1,1$.

Si un eterno viajero la atravesara en cualquier dirección, comprobaría al cabo de los siglos que los mismos volúmenes se repiten en el mismo desorden (que, repetido, sería un orden: el Orden).

J. L. Borges

La validez objetiva de todo saber empírico descansa en esto, y sólo en esto: que la realidad dada se ordene

'En el principio era el 'logos'. Frase con la que inicia el evangelio de San Juan y que resume la cosmovisión de la Antigüedad. Para el pensamiento griego el logos se entendía como palabra, lenguaje, pensamiento, pero también es razón, ley, regla, principio racional, razón suprema, conformidad con la ley, principio que impone orden, ... 
según categorías que son subjetivas en un sentido específico.

M. Weber

Recebido em 12.11.2009 Aceito em 05.03.2010

\section{Introducción}

El presente trabajo tiene como objetivo reflexionar acerca de los fundamentos filosóficos del orden bibliográfico. En este sentido, consideramos que las ideas y presupuestos desarrollados a lo largo de la historia en el ámbito de la organización bibliográfica, no son ajenos a otras manifestaciones culturales; por el contrario, estamos convencidos que existen fuertes vínculos entre el hacer bibliotecológico y los desarrollos y avances en la concepción del conocimiento. Desde este enfoque, la justificación de este trabajo consiste en tratar de mostrar que la organización es uno de los principios fundamentales de los que parte la existencia de la biblioteca, pues garantiza la libre circulación social de la información, la cual redunda en un mejor conocimiento de la realidad. Asimismo, concebimos el quehacer bibliotecológico como un producto humano comprometido con la investigación científica y cultural, que no sólo hunde sus raíces en el conocimiento socialmente acumulado (el cual siempre será un producto de los seres humanos); sino que también, está destinado a facilitar a quien lo desee y cuando lo necesite, los conocimientos e información requeridos en forma ágil y expedita.

El reflexionar sobre las bases filosóficas de organización de la información es regresar a los inicios del mundo bibliotecario y sus constructores. Sólo basta recordar que la gran biblioteca de la Antigüedad, la biblioteca de Alejandría, fue dirigida por grandes humanistas y científicos ${ }^{1}$, algunos de los cuales eran filósofos, tradición a la que después se unirían a través de la historia nombres como Casiodoro ${ }^{2}$, Alcuino ${ }^{3}$, Ockham ${ }^{4}$, Leibniz ${ }^{5}$, sólo por nombrar algunos. Asimismo, podemos

${ }^{1}$ Demetrio de Falero, Calímaco de Cirene, Zenódoto de Éfeso, Apolonio de Rodas, Eratóstenes, Aristófanes de Bizancio, Aristarco de Samotracia, Apolonio Eidographos.

2 Junto con Boecio e Isidoro de Sevilla se considera uno de los fundadores intelectuales del pensamiento medieval. A comienzos del siglo VI funda el monasterio de Vivarium y con sus obras De Institutione divinarum literarum y De Ortographia elabora un programa para que los monjes de su monasterio se dedicaran a la lectura y copia de textos "para servir a Dios". El Vivarium desapareció sin dejar huella, pero las órdenes monásticas posteriores continuaron ese proyecto, dando así lugar a una de las instituciones medievales más importantes: las bibliotecas monacales con sus copistas (GONZÁLEZ CASTRILLO, 2002, p. 102-103).

${ }^{3}$ Clérigo de origen inglés, consejero de Carlomagno y propulsor de las reformas en educación y cultura en general en el llamado renacimiento carolingio. Al final de su vida reunió una importante biblioteca.

${ }^{4}$ Filósofo nominalista de la edad media, nacido en Inglaterra. De acuerdo con el historiador soviético de la Lógica, N. I. Stiazhkin, la clasificación de las ciencias propuesta por Ockham en el siglo XIII "resultó ser muy firme. Basta decir que por ejemplo en el actual sistema decimal de clasificación de documentos, la sección "sistema científico" (índice 168) se divide en las subsecciones: "ciencias formales" (índice 168.51) y "ciencias reales" (índice 168.52), que exactamente corresponde a la división ockhamista de la ciencia en racionales y reales". (STIAZHKIN, 1967, p. 144). En la traducción al español del CDU el esquema correspondiente al 
recordar que los principios de clasificación bibliográfica tuvieron su fuente en clasificaciones de las ciencias realizadas por diferentes filósofos.

En esa línea de conexión entre las bibliotecas y la filosofía, Riaño Alonso señala que las bibliotecas en Grecia fueron una consecuencia directa del ejercicio y la práctica de la filosofía. De esta manera la Biblioteca de Alejandría no nació sólo por capricho, ocurrencia o deseo de prestigio de los Ptolomeos, ni tuvo por modelo los depósitos orientales de tablillas, sino que su origen estuvo ligado a una visión histórico-cultural concebida dentro de las escuelas filosóficas helenísticas (la Academia, el Liceo, la Estoa). La misión básica que se le confería era crear e irradiar el clima político y cultural del Helenismo. Es por eso que el modelo bibliotecario alejandrino, culminado por la figura de Calímaco de Cirene, tuvo tal éxito y se insertó profundamente en el paradigma bibliotecario, que se convirtió en el arquetipo de las centros bibliográficos que se continuaron erigiendo en el mundo grecorromano (RIAÑO ALONSO, 2005).

Nuestro objetivo en este texto es mostrar que la categoría de "orden", que crea el cosmos y la armonía, subyace en la base de la organización bibliográfica, como su fundamento último. Pero al mismo tiempo ese orden es otorgado, no dado a priori por el objeto de manera natural, sino a posteriori en función del sujeto de manera artificial. Lo anterior no significa que esa realidad creada sea relativa e incognoscible, sino humana; de la misma manera que el lenguaje y toda la cultura, que pueden ser estudiados con metodologías y enfoques específicos (hermenéutica, pragmatismo, tipos ideales, constructivismo, teoría crítica, entre otros) y en los que difícilmente se encontrarán leyes que las expliquen, pero que pueden ser conocidas a través de sentidos $y$ significados que nos conduzcan a su comprensión.

Cabe señalar que utilizamos los términos 'organización de la información' y 'organización bibliográfica' de manera indistinta. Su empleo responde a la diferenciación que se hace en el tiempo y a la evolución conceptual y terminológica que se establece en cada uno de los periodos históricos. La connotación permanece siendo la misma: en primer lugar la información, como ente ideal (abstracto) pero al mismo tiempo objetivado (materializado) en documentos que pueden ser de distinta naturaleza; y en segundo lugar las formas de descripción, como se le denomina y concibe en la actualidad a la materia prima del quehacer bibliotecológico, de esa información objetivada.

\section{En el inicio era el orden. El cosmos griego}

La cultura griega, que sigue abrevando nuestra cultura contemporánea en política, filosofía, literatura, arte, lenguaje, entre otros

168 es "El método científico" y la 168.51 es "Ciencias formales" y el 168.52 es "Ciencias empíricas".

5 Filósofo, matemático, lógico, jurista, político, fue uno de los grandes pensadores del siglo XVII y XVIII. Fue bibliotecario de la Casa de Brunswick en Hannover, donde desarrolló catálogos sistemáticos (ROSS, 1984). 
aspectos, también en el problema que nos ocupa en este trabajo, nos ayuda a encontrar un eslabón para comprender el fundamento filosófico de la organización bibliográfica. Dicho eslabón es la categoría de kosmos

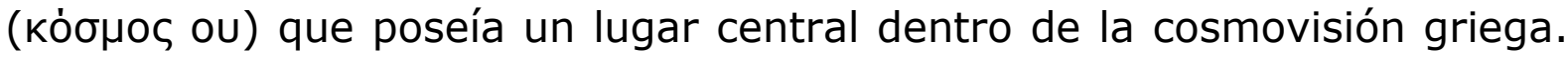
El término mencionado significa no solo universo, mundo, cielo, sino al mismo tiempo denota orden, organización, disciplina. Lo anterior porque el universo era para los griegos ante todo un universo ordenado, donde reinaba la armonía (ley, orden, justa proporción). Antes del cosmos sólo había caos. Podemos recordar el mito de Hesíodo de la cosmogonía, donde el Caos es lo que existe antes que nada, pero después deja su lugar a la primera y segunda generación de dioses. Con el cosmos constituido no había lugar para el caos, ya que uno y otro son antagónicos.

Ese orden presente en la naturaleza, en el arte, en la conducta humana existe porque está regido por el logos ( $\lambda$ óyos) que es Razón, ley, palabra, pensamiento entre otros muchos significados. Un fragmento de Heráclito describe esa cosmovisión griega donde reina el orden, la armonía, gracias a la dirección de un logos: "Cuando se escucha, no a mí, sino a la Razón (logos), es sabio convenir en que todas las cosas son una" (HERÁCLITO, 1987).

El orden regido por el logos es necesario, ya que el ser humano sólo puede vivir y orientarse en un mundo con orden, pues al adaptar las cosas a la regla impuesta por el logos, se hace posible, "saber lo que son las cosas ahora y siempre, captar lo que de eternas tenían, lo permanente en medio del cambio." (ZEA, 1993, p. 30-31) Más aún, "en la vida de cada individuo, "ibris" (úßpıৎ $\varepsilon \omega \varsigma$ ) el exceso, el pasarse de lo que es bueno y adecuado para el hombre, trae consigo la caída y exige el enderezamiento, la vuelta al equilibrio de la balanza, así, por extensión al universo, reina en todo la ley cósmica, la conservación de un equilibrio que impide el caos y la anarquía" (COPLESTON, 1986, p. 36).

Asimismo, durante el Medioevo la idea de un universo ordenado continuó, aunque con ropajes teo-ontológicos. La cosmovisión medieval tenía ahora como centro a Dios y éste pasaba a ser el ordenador, el que crea todo a la perfección; el logos griego dejó su lugar al Dios medieval. Para San Agustín y Santo Tomás el orden es una determinada relación recíproca de las partes; es una perfección que aparece como subordinación de lo inferior a lo superior, en donde existe una jerarquía ontológica.

En la filosofía de la Edad Media podemos encontrar la armonía del universo en la doctrina tomista de los llamados trascendentales del ente, que son la unidad, la verdad, la bondad y la belleza. De acuerdo con esta idea, todo ente es uno, verdadero, bueno y bello.

El ente es uno porque en relación a su ser no puede descomponerse en partes, lo único que se le opone, en cuando que es, es la nada, y la nada no existe, es decir, el ente tiene unidad en sí mismo (o es, o no es; pero como es, entonces es y punto). Al mismo tiempo, como es no contradictorio (es y no puede no ser), puede ser captado por el intelecto y por eso es verdadero, solo el ser es comprensible, el no ser es inconcebible. La verdad se entiende como la adecuación del ser con el 
intelecto. Simultáneamente el ente como uno y verdadero es captado por la voluntad y es apetecible, querido, en ese sentido es bueno. La Bondad se concibe como la adecuación del ser con la voluntad. Finalmente, como verdadero y bueno, el ente es bello.

Esa doctrina de los trascendentales nos muestra el optimismo y equilibrio del pensamiento medieval. El mundo es creado por Dios y como éste es no solamente omnipotente sino tiene todas las perfecciones, entre ellas la sabiduría y la bondad, por consiguiente, ese mundo también es inteligible, bueno y bello.

\section{El orden en la modernidad. La mirada hacia la razón y el conocimiento}

El mundo medieval dejó paso al mundo moderno, esto significó un cambio de cosmovisión en el que el centro era Dios, garante de la armonía en el mundo natural, de la posibilidad del conocimiento, del orden político y social, de la ética y la justicia; a una cosmovisión en el que la razón humana se alzaba con esas pretensiones cuasi divinas. La modernidad impuso a la razón como garante del orden y la armonía. Es por eso que si en la antigua Grecia las reflexiones giraban en torno a una ontología lógica; en la Edad Media, en torno a una ontología teológica; en la modernidad se volvió la mirada a reflexiones gnoseológicas, sobre el conocimiento, sobre cómo la razón conoce. Descartes, Leibniz, Bacon, Locke, Hume, Kant centran su interés primero en el aspecto gnoseológico más que ontológico porque había que fundamentar todo un modo de pensar de una época, basada en la razón humana.

El tránsito de la Edad Media a la Edad Moderna se empieza a dar desde los siglos XIV y XV con el movimiento que se denominó Renacimiento, pero se preparó casi un siglo antes con las luchas políticas entre el Papa y los Emperadores, levantamientos sociales disfrazados de herejías; cambios ideológicos y filosóficos como el despertar, con Roberto Grosseteste y Roger Bacon, de una metodología empírica y matemática. El desarrollo de esa nueva forma de investigación científica condujo a un progreso vertiginoso de la ciencia, principalmente de la Física y la Astronomía, conocido como revolución científica de los siglos XVI y XVII. Los nombres de Copérnico, Galileo, Tycho Brahe, Kepler y Newton están ligados a esa revolución, producto de observaciones, experimentos, mediciones que llevaron al descubrimiento de leyes y regularidades.

Al mismo tiempo que esos descubrimientos en las ciencias particulares, se dio la fundamentación filosófica de dicho proceso mediante la crítica a los conocimientos medievales, puramente especulativos, basados en lo escrito en textos de ciertos autores reconocidos como autoridades (Aristóteles, Tomás de Aquino, Padres de la Iglesia, Biblia) sin volverse a la realidad, a través de la justificación de la metodología empírica, inductiva, y matemática; así como en el énfasis del valor práctico del conocimiento frente al meramente contemplativo.

En esta línea cabe resaltar los pensamientos de Descartes, reconocido como el padre del pensamiento moderno, y de Francis Bacon, 
filósofo del método inductivo experimental. Ambos pensadores critican el conocimiento medieval, Descartes con su conocida duda metódica "no aceptar nada como verdadero, antes de haber obtenido la evidencia de que es así"; y Bacon con su crítica de los ídolos o prejuicios que impiden llegar al conocimiento, los ídolos de la tribu (idola tribus), propios de la especie humana que tiende a ver orden y causas universales; los ídolos de la caverna (idola specus) propios de la individualidad, que por gustos, intereses, educación introducen subjetividad en la visión del mundo; los ídolos del mercado (idola fori), que aparecen por el contacto entre los hombres y problemas lenguaje, causan confusiones, ya que "cuando los conceptos faltan, los suplen oportunamente las palabras"; y los ídolos del teatro (idola Theatri) que son los errores causados por pseudo científicos que a manera de actores proclaman sus falsas teorías en escuelas o universidades.

Con respecto al método que plantean estos filósofos, Descartes después de salir de su duda metódica al encontrar la verdad "clara y evidente" en el principio del cogito ergo sum, vuelve los ojos a las matemáticas como modelo de conocimiento, porque ellas proporcionan la necesidad, evidencia y claridad en sus conocimientos buscados. De esta manera, como señaló Galileo, las matemáticas son el lenguaje propio de la naturaleza, y por lo tanto el método para investigarla, sin su ayuda resulta imposible comprender una palabra de la naturaleza.

Por su parte, Francis Bacon propone un rechazo de la lógica aristotélica, que equivalía a la silogística, como método inadecuado para la investigación científica, y subraya la necesidad de encontrar un nuevo método de investigación de la naturaleza. Por ello denomina a su obra Novum Organum ya que la obra lógica de Aristóteles se conoce como Organon. Bacon fundamentó el inductivismo como un método experimental. Para ello era necesario reorientar la ciencia de su época hacia la naturaleza y los hechos, además de usar una metodología que consistió en una observación cuidadosa y completa de los hechos, que llama «historia natural y experimental», realizada según tablas de presencia, ausencia y comparación o grados.

Otra característica que el pensamiento moderno le otorgó al saber científico es la utilidad práctica del conocimiento teórico. Principalmente Bacon establece la estrecha relación entre la ciencia y el dominio de la naturaleza. "El conocimiento es poder" porque proporciona a los seres humanos la posibilidad de manipular la realidad a su deseo, y por supuesto, la voluntad se considera buena. Ella misma debe estar bajo el control del intelecto. De esta manera se abren los horizontes del progreso humano impulsado por el desarrollo científico.

Dentro de este contexto donde todo gira alrededor de la problemática gnoseológica, el interés también se centró en la clasificación de la ciencia. Aunque la clasificación de la ciencia es un tema específicamente moderno, ya desde la antigua Grecia con Aristóteles encontramos la preocupación por establecer una división del conocimiento filosófico. Según el Estagirita, la filosofía se divide en teórica, donde se busca la verdad; práctica, donde se estudia la acción dirigida a un 
objetivo; y poética, donde el objeto exterior es producido por un agente. A la lógica la ubica como un instrumento (organon). Posteriormente en la edad media aparece la clasificación de las ciencias ligada a la educación que se impartía en esa época y que incluía la enseñanza de las así llamadas siete artes liberales (propias de los hombres libres y que se oponían a las artes manuales). Dichas artes liberales se dividían en el Trivium (Gramática, Dialéctica, Retórica) y el Quadrivium (Aritmética, Geometría, Astronomía, Música).

Para Descartes el conocimiento científico se divide en forma de árbol, donde la raíz es la Filosofía, el tronco la Física; y las ramas son la Lingüística, la Economía, la Medicina, el Derecho, la Astronomía, la Geometría, y la Ética.

Para Francis Bacón las ciencias se clasifican según las facultades del hombre: razón, memoria e imaginación. A cada una de esas facultades le corresponden algunas disciplinas:

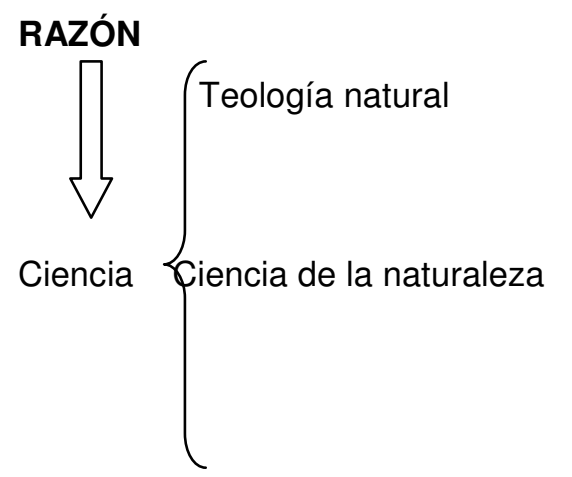

Ciencia del hombre Ética

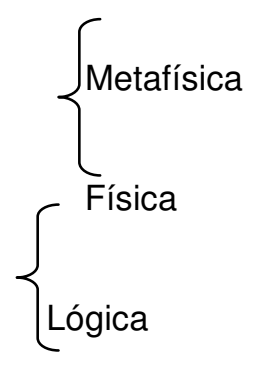

Ciencia de la sociedad

1 MEMORIA

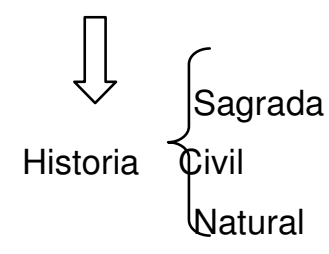

2 FANTASÍA

Poesia o Bellas Artes

Esta clasificación resultó ser muy importante porque sirvió como base para el sistema de clasificación de M. Dewey.

Otro gran pensador de la modernidad fue Leibniz, quien concibe a las ciencias como un continuum, cuyas divisiones son arbitrarias y sólo admisibles por comodidad. En su obra Nuevos ensayos sobre el entendimiento humano Leibniz expresa la idea de que el cuerpo entero de 
las ciencias puede ser considerado como el océano, que es continuo por todas partes y sin interrupción, aunque los hombres pueden concebir líneas en él y poner nombres a su comodidad. En este continuum, las ciencias son equipolentes y pueden organizarse de muchas maneras, según la función que ocupen en el todo o según otros criterios materiales o formales. Leibniz ha dejado no menos de veinte clasificaciones de las ciencias (LEIBNIZ, 1764, p. 247-250).

La idea de que no existe un "orden natural" en la clasificación de las ciencias también es importante en nuestro análisis porque como veremos más adelante, el orden dado al conocimiento científico es otorgado por el clasificador, cuestión que Borges pondría en evidencia en su "Torre de Babel", donde "los mismos volúmenes se repiten en el mismo desorden (que, repetido, sería un orden: el Orden)"

Durante la Ilustración las ideas de Bacón influyeron en la estructura y el orden que recibió la Enciclopédie ou Dictionnaire raisonné des sciences, des arts et des métiers [Diccionario razonado de las ciencias, de las artes y de los oficios]. Efectivamente, D'Lambert y Diderot, siguieron el proyecto baconiano, pues dieron a su obra un apartado de historia, otro para la filosofía y un tercero para las bellas artes. Así, cada uno de estos grandes grupos de ciencias y actividades humanas se subdividió en muchos otros apartados que configuran el árbol «de las ciencias, de las artes y de los oficios». Adicionalmente, la diferencia entre las enciclopedias antiguas (la Historia natural, de Plinio el Viejo, las Etimologías de san Isidoro, las Sumas de los escolásticos cristianos) y las modernas está en que aquéllas, persiguen conservar los conocimientos de una época; y éstas, difundirlos. ${ }^{6}$ Visto así, la Enciclopedia se presentó como un sistema de todos los conocimientos humanos; al ser éstos inabarcables, busca una manera de representarlos en síntesis.

El modelo científico desarrollado en la modernidad fue el de las ciencias naturales porque como ya indicamos, fueron la Física y la Astronomía en los siglos XVI y XVII las que se desarrollaron de manera vertiginosa. Después le tocó el turno a la Química en el siglo XVIII y a la Biología en el XIX, para que finalmente en el siglo XX se realizara una revolución en todas las ciencias naturales y las tecnologías, destacándose el desarrollo de la computación, telecomunicaciones y genética. De esta manera, teniendo como modelo de la ciencia a las ciencias naturales, nació el Positivismo, que exaltaba el uso del método científico empírico, matemático y lógico para descubrir leyes generales que permitieran explicar y predecir la realidad.

Fue hasta el siglo XIX, con excepción del pensamiento de G. Vico en el siglo XVIII, que las Ciencias Sociales y Humanas levantaron la mano para que se las tomara en cuenta como un cuerpo de conocimientos distinto a la de las ciencias naturales. De esta manera para los neokantianos W. Windelband se distinguen las ciencias nomotéticas (que

\footnotetext{
${ }^{6}$ La Enciclopedia se editó por primera vez entre los años 1751 y 1772, en París, Francia. Formada por 17 volúmenes editados por Denis Diderot y Jean Le Rond d'Alembert, su objetivo genérico fue difundir las ideas de la Ilustración francesa.
} 
descubren leyes) de las idiográficas (que buscan sentidos), y para $\mathrm{H}$. Rickert esa distinción equivale a las ciencias naturales y las ciencias de la cultura. De manera semejante, W. Dilthey divide a las ciencias en naturales, que pretenden explicar y siguen el modelo positivista, de las ciencias del espíritu, que tienden a comprender y cuyo método es la hermenéutica (interpretación para llegar al sentido de las acciones).

\section{La posmodernidad y el advenimiento del caos}

Fue dentro de este contexto donde apareció un nuevo movimiento que se ha denominado posmodernidad. En efecto, debido al desarrollo de las ciencias sociales y humanas; a cierta decepción en alcanzar un conocimiento universalmente verdadero ${ }^{7}$; al desencanto por las promesas incumplidas basadas en el poder de la razón ${ }^{8}$, el proyecto de la modernidad se tambaleó y surgió otra visión alternativa: la posmodernidad, la cual a su vez, también afectó el modo de organizar el conocimiento.

Es muy difícil definir un fenómeno tan complejo que abarca múltiples pensadores y esferas de la cultura como es la posmodernidad. Nos acercaremos a esta problemática siguiendo a Habermas (1989, p. 69 y ss; 1988, p. 19-36) y Beuchot (1996), quienes toman como punto de partida para su análisis de las diferentes escuelas posmodernas la posición que se adopta frente a la razón y al ideal de la Ilustración. De acuerdo con ese criterio, según Habermas se puede criticar la modernidad desde la izquierda o desde la derecha. En ambos casos se acepta algo del proyecto de la modernidad. Pero también se puede criticarla negándola totalmente.

En el caso de la crítica a la modernidad desde la izquierda, por un lado se aceptan algunos de sus logros, pero por otro, también se desea superar la razón meramente instrumental con otro tipo de razón, ya sea a través de la acción comunicativa (Habermas), la hermenéutica (Gadamer), o la pragmática (Appel). Por su parte, los críticos de la derecha o neoconservadores, aceptan la técnica, la razón instrumental; pero rechazan la cultura, la razón ética-utópica. Entre éstos emcontramos a C. Schmitt, Gottfried Benn, Arnold Gehlen, Daniel Bell. Finalmente, encontramos a los que los niegan por completo la modernidad y son críticos radicales de la razón; son los llamados antimodernos, y algunos de sus representantes son el "segundo" Heidegger, J. Derridá, G. Bataille, G. Deleuze, M. Foucault, F. Lyotard, G. Vattimo, R. Rorty. En esa misma línea de negar la modernidad pero con la propuesta de regresar a posiciones

\footnotetext{
7 Aparecieron limitaciones en las ciencias, incluso en aquellas que parecían las más sólidas. Por ejemplo en la Física, surgieron la teoría de la relatividad que echó por tierra la Física clásica; la física cuántica, donde parecen fallar algunas leyes lógicas; y el descubrimiento del llamado principio de indeterminación de Heisenberg. En matemáticas el teorema de Gödel acabó con las esperanzas de encontrar un sistema formal para la aritmética, donde todas las fórmulas universalmente verdaderas en ella sean teoremas (teorema de completud), ya que estableció que cualquier teoría axiomática no contradictora, en un lenguaje de primer orden adecuado para la teoría de números es esencialmente incompleta, es decir, siempre existirá un enunciado aritmético verdadero que no se puede demostrar en la teoría.

${ }^{8}$ A cambio del progreso hubo guerras mundiales, campos de concentración, dictaduras, hambre, problemas ecológicos.
} 
anteriores a la modernidad como el neoaristotelismo se encuentran algunos pensadores que son conocidos como paleoconservadores 0 premodernos, entre los que se pueden enumerar L. Strauss, R. Spaeman, A. MacIntyre; H. Jonas y S. Hauerwas. (BEUCHOT, 1996, p. 9)

La posmodernidad alcanza el problema de la organización del conocimiento en dos aspectos; el primero en cuanto se puede ver el sueño de la sociedad de la información o conocimiento como una vertiente posmoderna de derecha, y segundo como la introducción del relativismo, subjetivismo, y cierto "desorden" en la organización (uso de lenguaje libre, lógicas difusas) como influencia de una posmodernidad antimoderna.

\section{Bibliotecología contemporánea}

Aunque como hemos visto la práctica bibliotecaria se remonta a la antigüedad y la edad media, es en la edad moderna cuando se toma conciencia de esa práctica y finalmente se constituye como disciplina independiente. No es de extrañar que el primer manual conocido de Biblioteconomía, obra de Gabriel Naudé Advis pour dresser une bibliothèque fuera publicado en el siglo XVII, que la creación de los sistemas de clasificación aparecieran en el siglo XIX, M. Dewey se inspirara en la clasificación de las ciencias de Bacon, y la Biblioteconomía naciera bajo el modelo positivista de la ciencia. Todos esas circunstancias son un reflejo de que esta disciplina es una ciencia eminentemente moderna, es producto de la modernidad.

En efecto, si consideramos algunos de los valores que más se aprecian en Bibliotecología: el universalismo para compendiar todo el saber humano; el ideal de la Ilustración que predica que el conocimiento y la verdad sirven para ser mejores; la preocupación por la normalización, la organización y la sistematización; entonces descubrimos que esos mismos valores son distintivos y característicos de la modernidad.

Tomado en consideración todo lo enunciado anteriormente, podemos afirmar que en Bibliotecología rige el principio del orden, ya que se respetan los conceptos de organización, jerarquía y prioridad, posterioridad y sucesión, así como la estricta observancia de la norma fijada y su relación recíproca. El sustentar la organización documental en las relaciones de los documentos, sus contenidos y la representación simbólica que los ubica y conjunta, permite que se integren colecciones documentales que hagan explícito el significado de dichas relaciones, creando un núcleo de conocimientos acerca de los documentos que conforman la colección, logrando su relevancia dentro de la misma organización.

Ello conduce al bibliotecario al reconocimiento de la necesidad de utilizar la clasificación como herramienta de organización. La traducción de la clasificación como norma da por resultado al sistema de clasificación, en el cual, la representación documental se conforma y ordena a través de la asignación y empleo de símbolos. Entonces, el sistema clasificador tiene como finalidad, servir en la ordenación de los documentos mediante 
relaciones de interpretación que implican un proceso dirigido a, por un lado representar al documento y por otro a dotarlo de significado, con lo cual se pretende brindar coherencia a la diversidad documental, con el objeto de explicar los vínculos existentes entre los documentos que forman parte de la colección.

Gracias al sistema de clasificación se organiza el conocimiento en taxonomías o categorías. Las taxonomías representan mecanismos construidos para clasificar las cosas en una serie de grupos jerárquicos cuya función es facilitar la identificación, estudio y localización; así como también permiten la inclusión de estructuras y aplicaciones. Las estructuras se centran en los términos y sus relaciones, y las aplicaciones son las herramientas que facilitan su uso. Actualmente esto se traduce en el proceso que inicia con el análisis documental, orientado por las reglas de catalogación y los sistemas de clasificación documental que pretenden obtener y ofrecer representaciones del documento, su contenido y todas las posibles relaciones existentes.

La clasificación en bibliotecología contenida en el catálogo, tiene como finalidad definir un universo cuya primera instancia permita determinar si un registro en particular puede incluirse o no como parte del universo de documentos, además de que crea un espacio en donde se establecen relaciones lógicas entre los elementos de los registros pertenecientes a ese universo y sirve de base para la organización física de los documentos y sus representaciones.

Así como la organización física de los documentos tiene lugar, encontramos a la organización temática o de contenido, la cual para ser visible, manipulable y conjuntable desarrolla el registro documental. Dicho registro se basa en los principios de catalogación, tarea mediante la cual se describe a los elementos físicos y temáticos que caracterizan a un documento, mismos que nombran a cada una de las partes que lo constituyen y establecen el orden que deben guardar dentro de dicho registro. El orden y las relaciones entre los elementos de un registro, dotan de significado al documento, su información y contenidos, lo que permite la relación de los elementos entre registros.

Ahora bien, la reunión de múltiples registros dan como resultado la existencia del catálogo, que en su estructura global, manifiesta las relaciones entre registros documentales, documentos, agrupación y ubicación espacial; haciendo evidente su propia forma de ordenación que es guiada por la normalización en su continuo crecimiento y refleja el orden bibliográfico-documental de la biblioteca.

Así pues, el núcleo de conocimientos se verá representado por el catálogo de la biblioteca ${ }^{9}$, en el cual se observa la conjunción de los tres tipos de orden hasta ahora desarrollados -físico, temático y de registro-, que lo convierten en la puerta de acceso a la información contenida en los documentos conjuntados en el acervo.

\footnotetext{
${ }^{9}$ Específicamente en el catálogo electrónico, se visualiza la mayor cantidad de relaciones posibles entre los contenidos temáticos de los documentos gracias a los sistemas de pre-coordinación y pos-coordinación que en el subsisten.
} 
Aunque en nuestros días todavía los grandes sistemas de clasificación y las normas catalográficas ${ }^{10}$, que inicialmente fueron adoptadas como respuesta a la sobre carga de información generada debido al descubrimiento de la imprenta, siguen resolviendo los problemas de acomodo temático de los documentos, y pueden seguir siendo considerados como eficientes instrumentos de sistematización de los contenidos documentales y del conocimiento, actualmente existen otras propuestas para identificar, representar y tener acceso a los documentos, principalmente debido a la aparición de documentos electrónicos. Por ejemplo el modelo propuesto por el Dublín Core para metadatos y los lineamientos de descripción dados en las RDA, los FRBR. ${ }^{11}$ Sin embargo no es difícil constatar que el principio y categoría del orden se sigue manteniendo en esas nuevas propuestas.

\section{Consideraciones finales}

El análisis de lo hasta aquí expuesto nos permite afirmar que ha semejanza del mito de Hesíodo sobre la teogonía, el caos informacional deja su lugar al "cosmos documental", donde rige un orden, gracias al cual es posible orientarse, moverse y actuar en ese cosmos. La particularidad de eso que hemos denominado cosmos documental es que es construido por la actividad del bibliotecario, es pues una creación humana y por consecuencia cultural. En otros escritos hemos identificado el sistema de organización documental como objeto de estudio de la bibliotecología (RENDÓN ROJAS, 2005) y conectando esa idea con esta reflexión sobre las bases de la organización documental, podemos decir que el bibliotecario con su acción construye ese sistema; el cual no es un ente natural dado (piedra, planeta, elemento químico por ejemplo) al que hay que interpretar para dotarlo de sentido; sino que incluso su misma existencia es mediata.

El nombrar (representar) el ente informativo-documental, el crear una organización, el realizar lo necesario para brindar el acceso a éste, es construir el mundo informativo documental propio de la bibliotecología; un mundo como todo lo humano, lleno de sentidos, pero sentidos de segundo orden, es decir, sentidos no del mundo natural, sino sentidos de un mundo cultural que a su vez es interpretado. Por ello la bibliotecología no es una ciencia natural, sino siguiendo la terminología de Dilthey, una ciencia del espíritu.

Si tomamos por ejemplo la clasificación que se realiza en la organización documental, encontramos que la clasificación es una

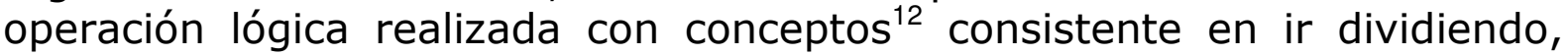
con base en ciertos criterios, el volumen del concepto inicial mediante la

${ }^{10}$ El sistema de clasificación de Dewey, la clasificación decimal universal (CDU), la clasificación del Congreso (LC); las Reglas de catalogación anglo-americanas segunda edición (RCAA2).

11 Resource Description and Access (RDA); Functional Requirements for Bibliographic Records (FRBR).

12 Entendemos por concepto a la forma de pensamiento que con base en ciertas características identifica y distingue a ciertos objetos dentro de un universo, y los agrupa en un conjunto. Éste último se denomina volumen del concepto. 
formación de subclases o subconjuntos sucesivos. Dichos criterios pueden ser propiedades inherentes al objeto, presentes como características esenciales; o pueden ser características que por sí mismas no son relevantes para el objeto en sí, pero que se emplean como criterios para realizar una tarea determina. En el primer caso se puede decir que es una clasificación natural basada en atributos esenciales de los objetos, como es el caso de la clasificación de los elementos de acuerdo con la Tabla periódica de Mendeliev, donde el peso y número atómico, determinan el lugar y propiedades de los elementos; o las clasificaciones taxonómicas de los seres vivos en biología, donde la anatomía comparada y la evolución de las especies orientan la clasificación. En el segundo caso, los criterios de la clasificación se basan en características que se eligen porque son convenientes para alcanzar un fin, como sería el clasificar a los alumnos por estatura, peso, o las palabras por orden alfabético. En este último caso se tienen clasificaciones artificiales.

El hecho de crear un mundo informativo documental artificial, con clasificaciones arbitrarias, ha llevado a pensar, dentro del contexto posmodernista, a que en la biblioteca y su cosmos reina la subjetividad total, la arbitrariedad absoluta. Sin embargo debemos aclarar que lo artificial se contrapone a lo natural, en el sentido de su génesis, depende de la acción de un sujeto y lo natural no; pero no es sinónimo de relativismo e incapacidad de ser estudiado y conocido. En primer lugar, el sistema creado, tiene sus regularidades en cuanto ente existente. En segundo lugar, la misma construcción del sistema informativo documental no es una creación ex nihilo, sino precisamente una construcción que utiliza materia prima inicial; y esa materia prima: información, documentos, usuarios poseen unas características que son los soportes que le dan objetividad al orden creado. Toda interpretación, aunque depende del sujeto, debe respetar al texto, en este caso esa materia prima de la que hablamos líneas arriba. Ese atributo de ser una realidad objetiva y necesaria es a lo que hemos llamado "fundamentación ontológica de la bibliotecología", la cual hemos desarrollado previamente (RENDÓN ROJAS, 2005, p. 54-78) y por consecuencia demostrado que el cosmos bibliotecológico, posee su ontología propia, abstracta y humana; y al mismo tiempo no es algo inventado por un voluntarismo absoluto, sino descansa en una ontología de primer nivel, anclado en características reales del ser humano, que englobamos con el término "ser informacional", ya que el ser humano por su estructura ontológica para realizar su ser necesita crear, consumir, transformar información en cuanto es un ser dialógico, hermenéutico, histórico, simbólico, político, social, económico, racional.

De esta manera, si queremos seguir con la metáfora de la teogonía hesiódica, podemos concluir que en el principio es el ser informacional, de este nace el cosmos documental regido por el orden, nacimiento mediado por la actividad del sujeto; y finalmente, ese orden posee sus propias sus propias regularidades, que pueden ser estudiadas. 


\section{Bibliografía}

BEUCHOT, M. Posmodernidad, hermenéutica y analogía. México: Miguel Ángel Porrúa: Librero-Editor; Universidad Intercontinental, 1996

COPLESTON, F. Historia de la filosofía : Grecia y Roma. Barcelona: Ariel, 1986.

GONZÁLEZ CASTRILLO, R. Oposiciones a bibliotecas y archivos. (Escalas de Facultativos y de Ayudantes). Temario Básico. Madrid: Editorial Complutense, 2002.

HABERMAS, J. El discurso filosófico de la modernidad. Madrid: Taurus, 1989.

HABERMAS, J. La modernidad un proyecto incompleto. En: FOSTER, H. et al. La Posmodernidad. México: Kairós-Colofón, 1988. p. 19-36.

HERACLITO. Fragmentos. En: KIRK, G. S.; RAVEN, J. E.; SCHOFIELD, Y M. S. Los filósofos presocráticos: historia crítica con selección de textos. Madrid: Gredos, 1987.

LEIBNIZ, G. W. New Enssays on Human Understanding. En: BENNETT, J. Early moder texts. 1764. Disponible en: <http://www.earlymoderntexts.com/pdfbits/ne43.pdf>. Consultado en: septiembre de 2009.

NAUDÉ, G. Advis pour dresser une bibliothèque. Paris: Klincksieck, [1627] 2008.

RENDÓN ROJAS, M. A. Bases teóricas y filosóficas de la bibliotecología. 2a ed. México: UNAM; Centro Universitario de Investigaciones Bibliotecológicas, 2005.

RIAÑO ALONSO, J. J. Naturaleza de la Antigua Biblioteca de Alejandría. España: Trea, 2005.

ROSS, G. M. Leibniz. Oxford: Oxford University Press, 1984.

STIAZHKIN, H. I. Formirovanie matematicheskoi lóguiki (La formación de la lógica matemática). Moscú: Nauka, 1967.

ZEA, L. Introducción a la filosofí: la conciencia del hombre en la filosofía. México: UNAM, 1993. 